\title{
COMPARAÇÃO DOS EFEITOS DA IMERSÃO EM ÁGUA FRIA COM APLICAÇÃO DE PLACEBO NA FORÇA
}

Gabriela Carrion Caldeira Ribeiro ${ }^{1}$, Caio Ferreira Ripper $^{1}$, Jaqueline Santos Silva ${ }^{1,2}$, Franciele Marques Vanderlei ${ }^{1,3}$, Jéssica Kirsch Michelleti ${ }^{1,4}$, Aryane Flauzino Machado ${ }^{1,4}$, Larissa Rodrigues Souto $^{1,2}$, Malu Siqueira dos Santos ${ }^{1,2}$, Gabriela de Carvalho ${ }^{1}$, Jayme Netto Junior ${ }^{1,3}$, Carlos Marcelo Pastre ${ }^{1,3}$

${ }^{1}$ Universidade Estadual Paulista - UNESP, Curso de Fisioterapia, ${ }^{1,2}$ Mestrado em Fisioterapia, ${ }^{1,3}$ Departamento de Fisioterapia, Laboratório de Fisioterapia Desportiva-LAFIDE, ${ }^{1,4}$ Doutorado em Fisioterapia. Presidente Prudente, SP. Email: pastre@fct.unesp.br

\section{RESUMO}

Introdução: A imersão em água fria (IAF) é uma modalidade popular no meio esportivo, utilizada com fins de minimizar os danos fisiológicos pós-exercício e assim, otimizar os níveis de recuperação. Esta modalidade consiste na imersão de partes do corpo em água a uma temperatura baixa $\left(15^{\circ} \mathrm{C}\right)$. Neste cenário, o efeito placebo também vem ganhando destaque. Objetivos: Analisar e comparar os efeitos da IAF quando aplicada de forma sistemática, com a condição placebo na variável força. Métodos: $O$ estudo teve design cross over. A amostra foi composta por 10 atletas de alto rendimento, do sexo masculino, que foram alocados de forma randomizada em dois grupos: grupo placebo, submetido a uma intervenção por 15 minutos a $27^{\circ} \mathrm{C} \pm 1$ 으 $\mathrm{C}$ e grupo experimental submetidos a imersão em água fria (IAF) por 15 minutos a $13^{\circ} \mathrm{C} \pm 1$ 으. A avaliação da função muscular foi medida por meio da contração voluntária máxima, no dinamômetro isocinético. Resultados: Não foi observada diferença estatística para o pico de torque e torque total entre os grupos analisados antes e após o período de intervenção. Conclusão: Foi possível concluir que a variável força, não sofreu alterações significativas nas medidas pré e pós intervenção de IAF e placebo, na população analisada.

Palavras-chave: Crioterapia. Placebo. Força. Atletas.

\section{COMPARISON OF THE EFFECTS OF IMMERSION IN COLD WATER WITH PLACEBO APPLICATION IN STRENGTH}

\begin{abstract}
Introduction: The cold water immersion (CWI) is a popular mode in sports, used for purposes of minimizing the physiological damage after exercise and thus optimize recovery levels. This method consists in immersing parts of the body in water at a low temperature $\left(15^{\circ} \mathrm{C}\right)$. In this scenario, the placebo effect is also gaining prominence.Objectives: Analyze and compare the effects of the $\mathrm{CWI}$ when applied systematically, with the placebo condition in variable strength. Methods: The study had design cross over. The sample was composed of 10 high-performance athletes, male, which were allocated randomly into two groups: placebo group, subjected to an intervention for 15 minutes at $27^{\circ} \mathrm{C} \pm 1^{\circ} \mathrm{C}$ and sexperimental group subjected to immersion in cold water (CWI) for 15 minutes at $13^{\circ} \mathrm{C} \pm 1^{\circ} \mathrm{C}$. The evaluation of muscle function was measured by maximal voluntary contraction, in the isokinetic dynamometer. Results: There was no statistical difference in peak torque and total torque between the groups analyzed before and after the intervention period. Conclusion: It was concluded that the variable force, has not changed significantly inthe pre and post intervention $\mathrm{CWI}$ and placebo in the study population.
\end{abstract}

Key Words: Criotherapy. Placebo. Strength. Athletes. 


\section{INTRODUÇÃO}

As estratégias de recuperação pós-esforço representam aspecto importante no desempenho de atletas, pois reestabelecem os sistemas do corpo a sua condição basal, a homeostase, prevenindo assim instalações de lesões ${ }^{1}$. Entre as estratégias de recuperação após esforço de alta intensidade, a imersão em água fria, vem ganhando destaque. A imersão em água fria (IAF) consiste em uma modalidade de imersão de partes do corpo em água com temperatura baixa, igual ou inferior a $15^{\circ} \mathrm{C}^{2}$. Essa modalidade de resfriamento do tecido muscular tem sido realizada há décadas, a fim de obter objetivos terapêuticos específicos. Entre os objetivos destacam-se alterações no metabolismo, na condução nervosa e alteração da percepção da dor ${ }^{3}$. É uma modalidade popular no meio esportivo, utilizada com fins de minimizar os danos fisiológicos pós-exercício e assim otimizar os níveis de recuperação. Os efeitos da aplicação de IAF no músculo são bastante conhecidos, entre os quais ressalta-se a vasoconstrição, diminuição da velocidade de condução nervosa e diminuição da contratilidade muscular ${ }^{4}$. Nesse sentindo, estudos demonstraram que o resfriamento também pode afetar a força máxima e a velocidade de condução ${ }^{5}$.

No estudo de Cornwall et al $^{3}$ composto por 30 participantes saudáveis e fisicamente ativos, de ambos os sexos foi investigado o efeito do aquecimento e resfriamento na força máxima voluntaria e na taxa de desenvolvimento da força no punho em 3 condições distintas, imersão em água a $40^{\circ} \mathrm{C}$, IAF a $10^{\circ} \mathrm{C}$ e um grupo controle. Como resultado, foi observado que após a IAF a taxa de força e desenvolvimento de força diminuiu, enquanto que o tempo para atingir o pico de desenvolvimento da força em homens aumentou e nas mulheres somente o tempo para atingir o desenvolvimento do pico de força mostrou-se alterado, pois também aumentou.

Howard et al $^{4}$, realizou um estudo em uma população de estudantes universitários, do sexo masculino e fisicamente ativos, onde examinaram os efeitos da IAF sobre a força isocinética e isométrica e concluíram que a imersão à $12^{\circ} \mathrm{C}$ por 45 minutos diminuiu significativamente o pico de torque isocinético, porém não alterando a força. Davies e Young et $\mathrm{al}^{3}$, também realizaram a imersão por 30 minutos em água fria e termoneutra e evidenciaram resultados ligeiramente diferentes. Alta temperatura foi responsável por diminuição no tempo para atingir o pico de força, enquanto a menor temperatura foi responsável por efeito foi oposto.

A partir do exposto, IAF vêm sendo bastante utilizada e citada na questão da recuperação pós-exercício. Porém, existem lacunas no que se refere às respostas referentes ao efeito psicológico. Nesse cenário, um dos efeitos que vem sendo discutido, é o efeito placebo. $\mathrm{O}$ efeito placebo consiste em respostas fisiológicas ou psicológicas que são resultados de intervenções terapêuticas ${ }^{6}$. Por definição, esse efeito é privado de propriedades terapêuticas da condição a ser tratada ${ }^{7}$. Portanto, o efeito placebo é uma manifestação a ser elaborada para simular a terapia médica, acreditando não apresentar nenhum efeito específico sobre determinada doença ${ }^{8}$.

Assim, a necessidade de estudos referentes à variável força após estratégias de recuperação pós-esforço merece destaque, a fim de elucidar as lacunas existentes. Portanto, tendo em vista a importância sobre os mecanismos e respostas de recuperação, o presente estudo tem como objetivo demonstrar os resultados da variável pico de torque na imersão em água fria e placebo.

\section{MÉTODOS}

População

Trata-se de um ensaio clínico randomizado cruzado. A casuística do estudo foi composta por 10 atletas de alto rendimento do sexo masculino praticantes da modalidade de atletismo com idade entre 18 e 30 anos. O número de voluntários selecionado garante um poder de teste acima de $80 \%$ com nível de significância de $5 \%$. 
Foram incluídos no estudo atletas de alto rendimento. Os critérios de exclusão foram quaisquer tipos de lesão músculo esquelética antes e durante os períodos de coleta.

Os voluntários foram devidamente informados sobre os procedimentos e objetivos deste estudo, e após concordarem, assinaram um termo de consentimento livre e esclarecido passando a fazer parte efetivamente do mesmo. Todos os procedimentos utilizados neste trabalho foram submetidos ao Comitê de Ética em Pesquisa da Universidade Estadual Paulista - FCT/UNESP por meio da Plataforma Brasil. Número do protocolo: 44591115.0.0000.5402.

Intervenções de recuperação

Os voluntários foram alocados de forma randomizada em dois grupos de estudo: um grupo placebo (GP; $n=10$ ), foi submetido a uma intervenção recuperativa considerada placebo por 15 minutos a temperatura de $27 \stackrel{\circ}{\circ} \mathrm{C} \pm 1$ 으, o grupo experimental ( $G E, n=10)$, foi submetido a uma intervenção recuperativa por meio da IAF por 15 minutos a temperatura de $13^{\circ} \mathrm{C} \pm 1$ 으.

Para o GE a temperatura da água foi mantida por meio da colocação de gelo com medições realizadas por meio de um termômetro digital (Incoterm, Porto Alegre, Brasil) a cada 5 minutos, já a temperatura da água do GP foi considerada termo-neutra e também foram realizadas mensurações a cada 5 minutos. O protocolo de IAF utilizado nesse estudo foi baseado em um estudo anterior ${ }^{9}$, à $14 \stackrel{\text { ㅇ }}{ }$ por 15 minutos. Para facilitar o efeito placebo, foi adicionado à água à vista do voluntário imediatamente antes da imersão uma solução de limpeza de pele com pH balanceado (Cetaphil, Gentle Skin Cleanser, Australia) (Tabela 1).

Tabela 01. Distribuição dos grupos estudados.

\begin{tabular}{|c|c|c|}
\hline Grupos & Intervenção & Tempo \\
\hline Grupo Placebo (GP) & $\begin{array}{c}\text { Água termo-neutra a } \\
27 \text { o } \mathrm{C} \pm 1^{\circ} \mathrm{C} \text { com adição de } \\
\text { solução específica [Cetaphil] }\end{array}$ & 15 minutos \\
\hline Grupo Experimental (GE) & $\begin{array}{l}\text { IAF à temperatura de } \\
13^{\circ} \mathrm{C} \pm 1{ }^{\circ} \mathrm{C}\end{array}$ & 15 minutos \\
\hline
\end{tabular}

Para eliminar possível viés, os voluntários do GP foram levados a acreditar que a imersão em água termo-neutra com adição da solução de limpeza de pele é benéfica na recuperação pósexercício tanto quanto a IAF ${ }^{10}$.

Delineamento do estudo

A coleta de dados foi realizada na pista de atletismo "Mário Covas" e no Centro de Estudos e Atendimento de Fisioterapia e Reabilitação da Universidade Estadual Paulista (FCT/UNESP) campus de Presidente Prudente, nos horários das 8h00min às 18h00min dependendo do dia do treinamento, e todos os protocolos serão realizados conforme as condições climáticas do dia, portanto os valores da temperatura e da umidade relativa do ar serão mensurados nos dias de coleta.

Para realização deste estudo os voluntários foram submetidos a um ensaio composto por três etapas. Na primeira etapa foi determinada a condição basal do voluntário. Primeiramente foram coletadas informações pessoais como idade, tempo de treinamento e melhor resultado obtido nas competições, além de serem avaliados os parâmetros antropométricos dos voluntários (peso e estatura) ${ }^{11}$, e em seguida foi mensurada a pressão arterial de forma indireta ${ }^{12}$. 0 voluntário realizou a avaliação da função muscular por meio da contração voluntária máxima ${ }^{13}$.

Após a realização do teste inicial de força, foi dado início a segunda etapa do protocolo onde os voluntários iniciaram o período de treinamento, caracterizado por treinos periodizados estruturais, metabólicos e recuperativos composto por atividades de aquecimento, coordenação, agilidade, força muscular e potência com uma frequência de seis vezes por semana por seis 
semanas. Durante esse período, quatro vezes durante a semana, ao final do treino físico os participantes foram submetidos à intervenção randomizada inicialmente, sendo duas semanas na IAF, duas semanas na condição placebo e duas semanas na condição controle.

Com o término das duas semanas do protocolo, os grupos foram trocados em relação à intervenção realizada, caracterizando assim um design cross-over, ou seja, os participantes que nas duas primeiras semanas realizaram a intervenção por IAF, em seguida (3a e 4a semanas) realizarão a intervenção placebo e dependendo da randomização que foi realizada

\section{TREINAMENTOS}

As coletas foram realizadas em um período de treinamento denominado base em que é considerado um dos mais importantes da temporada e que tem característica de preparação física do atleta. A base foi realizada no início da temporada após o período de transição dos atletas (férias) e teve duração de seis semanas.

Durante esse período, o treinamento foi realizado seis vezes na semana por seis semanas, lembrando que durante quatro vezes na semana foi realizada a intervenção sendo dividida em dois blocos, anteriormente randomizadas: duas semanas de intervenção por IAF e duas semanas de intervenção por placebo. As atividades que foram realizadas nos dias dos protocolos durante esse período dependeram do objetivo do treinamento, no entanto durante esse período da base a ênfase dada é para o volume de treino e não para a intensidade. De modo geral, foram realizados treinos de musculação para membros superiores, membros inferiores e tronco, treino composto por atividades de coordenação, agilidade e potência muscular/tiros, sendo trabalhadas as capacidades aeróbicas e anaeróbicas dos voluntários.

\section{CONTRAÇÃO VOLUNTÁRIA MÁXIMA}

Para avaliação da função muscular, o voluntário foi posicionado com o membro inferior dominante no dinamômetro isocinético Biodex System Pro (Biodex Medical System, Shirley-NY, USA). Conforme protocolo sugerido por Baroni et al. ${ }^{13}$, anteriormente a avaliação, o voluntário será submetido a um aquecimento, que consistirá em dez repetições de contração concêntrica de flexo-extensão de joelho a 180 \%/s em toda amplitude de movimento.

A função muscular foi avaliada por meio do maior valor de torque obtido entre três repetições de cinco segundos de contração voluntária máxima a 60 de flexão de joelho (com 0응 correspondendo à máxima extensão). Um intervalo de dois minutos entre as repetições foi administrado, a fim de minimizar possíveis efeitos de fadiga. O voluntário foi instruído a realizar sua performance máxima de força e foi encorajado verbalmente pelo pesquisador em cada contração voluntária máxima.

\section{Análise Estatística}

Para análise dos dados do perfil da população foi utilizado o método estatístico descritivo e os resultados foram apresentados com valores de médias, desvios padrão, mediana e intervalo de confiança. A normalidade dos dados foi avaliada por meio do teste de Shapiro-Wills. Para comparação dentro dos grupos foi utilizado teste $t$ pareado para dados normais e teste de Wilcoxon para dados não normais e para a comparação entre os grupos foi utilizado teste $t$ não pareado para dados normais e Mann-Whitney para dados não normais. O nível de significância foi estabelecido em $\mathrm{p} \leq 0,05$ para todos os testes.

\section{RESULTADOS}

Não foi observada diferença estatística para pico de torque e torque total, entre os grupos analisados antes e após o período de intervenção. Os valores estão demonstrados na tabela 2.

A tabela 2 demonstra os valores de média, desvio padrão, mediana e intervalo de confiança da variável força antes e após aplicação sistemática de IAF e placebo. Para o grupo IAF, os valores 
basais de média, desvio padrão, mediana e intervalo de confiança para pico de torque foram respectivamente $348,37 \pm 66,12(352,00)[293,08-403,67]$ no momento basal. Por outro lado, os valores do momento final correspondentes foram respectivamente $336,75 \pm 49,55(315,50)[295,3$ 378,18]. Para o grupo placebo, os valores de momento basal foram $347,12 \pm 47,78(344,00)$ $[307,17-387,08]$. Ainda para o grupo placebo na variável pico de torque, os valores no momento final foram 317,75 $\pm 57,92(323,00)[269,32-366,18]$.

Para a variável torque total, o grupo IAF demonstrou valores basais equivalentes a $344,37 \pm$ $66,92(351,25)[288,41-400,34]$. Enquanto os valores de momento final foram $334,62 \pm 50,04$ $(314,85)[292,78-376,47]$. Por fim, o grupo placebo apresentou os valores basais de $345,22 \pm$ $47,27(343,35)[305,70-384,75]$. E valores finais de 314,41 $\pm 58,72(321,30)[265,31-363,52]$. Referentes a média, desvio padrão, mediana e intervalo de confiança respectivamente.

Tabela 02. Média, desvio padrão, mediana e intervalo de confiança ( $p$ ) das variáveis clínicas (contração isométrica voluntária máxima) antes e após a aplicação sistemática de IAF e placebo.

\begin{tabular}{|c|c|c|c|}
\hline Variáveis & Momentos & IAF & Placebo \\
\hline \multirow{2}{*}{ Pico de torque } & Basal & $\begin{array}{c}348,37 \pm 66,12 \\
(352,00) \\
{[293,08-403,67]}\end{array}$ & $\begin{array}{c}347,12 \pm 47,78 \\
(344,00) \\
{[307,17-387,08]}\end{array}$ \\
\hline & Final & $\begin{array}{c}336,75 \pm 49,55 \\
(315,50) \\
{[295,32-378,18]}\end{array}$ & $\begin{array}{c}317,75 \pm 57,92 \\
(323,00) \\
{[269,32-366,18]}\end{array}$ \\
\hline \multirow{2}{*}{ Torque Total } & Basal & $\begin{array}{c}344,37 \pm 66,92 \\
(351,25) \\
{[288,41-400,34]}\end{array}$ & $\begin{array}{c}345,22 \pm 47,27 \\
(343,35) \\
{[305,70-384,75]}\end{array}$ \\
\hline & Final & $\begin{array}{c}334,62 \pm 50,04 \\
(314,85) \\
{[292,78-376,47]}\end{array}$ & $\begin{array}{c}314,41 \pm 58,72 \\
(321,30) \\
{[265,31-363,52]}\end{array}$ \\
\hline
\end{tabular}

\section{DISCUSSÃO}

O presente estudo teve como objetivo avaliar o pico de torque e o torque total após a estratégia de recuperação em IAF e placebo em atletas de alto rendimento. Contudo, os achados do estudo demonstram que não houve diferenças estatísticas entre os grupos experimental (IAF) e placebo nos períodos analisados antes e após intervenção.

De acordo com achados na literatura, o estudo de Broatch et $\mathrm{AL}^{10}$ realizado com 30 participantes, do sexo masculino, realizaram uma sessão de treinamento intervalado de alta intensidade e analisaram a contração isométrica voluntária máxima. Após o treinamento os participantes foram alocados para 3 grupos: IAF $\left(10^{\circ} \mathrm{C}\right)$, placebo $\left(34^{\circ} \mathrm{C}\right)$ e controle $\left(34^{\circ} \mathrm{C}\right)$ por 15 minutos. Para facilitar a condição placebo, foi adicionado uma solução de limpeza de pele na água do grupo placebo. A principal conclusão deste estudo foi que o grupo placebo obteve uma melhor recuperação da força muscular do quadríceps quando comparado com o grupo controle, porém o resultado do grupo de IAF foi semelhante ao placebo. Além disso, o grupo placebo mostrou-se melhor na recuperação da força muscular após $48 \mathrm{~h}$ quando comparado com o grupo IAF. Tal fato, pode ser atribuído a uma melhora da percepção da dor e prontidão para o exercício, sugerindo a hipótese de que os benefícios fisiológicos da condição IAF, esteja relacionada parcialmente com o efeito placebo.

Estudo de Bailey et al ${ }^{2}$, teve como objetivo investigar os efeitos da IAF em índices de lesão muscular após uma sessão de exercício intermitente prolongado, concluiu que a IAF (10 minutos a $10^{\circ} \mathrm{C}$ ) aplicada logo após o exercício reduziu a sensação de dor no músculo, porém houve decréscimo na contração isométrica voluntaria máxima após a imersão quando comparado 
com o grupo controle, esse resultado pode ter ocorrido devido a uma lesão no músculo, disfunção ou até mesmo uma dor que não foi bem relatada.

Vale ressaltar, que os atletas do presente estudo, já eram adeptos da utilização de IAF. Neste caso, a familiarização da técnica pode ter sido um fator que influenciou diretamente os resultados. Além disso, pode ter havido falhas quanto a eficácia da maneira de levar os participantes a acreditarem na nova proposta de recuperação por meio da adição do óleo corporal à água termoneutra. Além das limitações destacadas, o baixo número da amostra também pode ter interferido nos resultados. Logo, a elaboração de novos estudos faz-se necessária para preencher as lacunas existentes sobre o tema.

\section{CONCLUSÃO}

Com base nos dados do presente estudo conclui-se que não houve diferença estatisticamente significante no pico de torque e torque total entre as intervenções nos grupos IAF e placebo.

\section{REFERÊNCIAS}

Pastre CM, Bastos FN, Netto Junior J, Vanderlei LCM, Hoshi RA. Metodos de Recuperacao Posexercicio: uma Revisao Sistematica. Revista Brasileira de Medicina do Esporte 2009 15(2): 138-144. https://doi.org/10.1590/S1517-86922009000200012

Bailey DM, Erith SJ, Griffin PJ, Dowson A, Brewer DS, Gant N, et al. Influence of cold-water immersion on indices of muscle damage followind proloned intermittente shuttle running. J Sports Sci. 2007; 25(11):1163-70. https://doi.org/10.1080/02640410600982659.

Cornwall MW. Effect of temperature on muscle force and rate of muscle force production in men and woman. Journal of Orthopaedic \& Sports Physical Therapy 1994 20: 74-80. https://doi.org/10.2519/jospt.1994.20.2.74.

Dixon PG, Kraemer WJ, Volek JS, Howard RL, Gomez AL, Comstock BA, Dunn-Lewis C, Fragala MS, Hooper DR, Hakkinen K, Mareshthe CM. Impact of cold water immersion on power production in the vertical jump and the benefits of dynamic exercise warm-up.The Journal of Strength and Conditioning Research 2010 24(12): 3313-3317. https://doi.org/10.1519/JSC.0b013e3181f212e2.

Kubo K, Kanehisa H, Fukunaga T. Effects of cold and hot water immersion on the mechanical properties of human muscle and tendon in vivo. Clinical Biomechanics(Bristol, Avon) 2005 20(3):291-300. https://doi.org/10.1016/i.clinbiomech.2004.11.005

Geers AL, Fowler SL, Wellman JA, Helfer SG, Close S, France CR. Prior experience with a pain stimulus as a predictor of placebo analgesia. Journal of behavioral medicine 2015 38(1): 136-142. https://doi.org/10.1007/s10865-014-9586-1.

Pollo A, Carlino E, Benedetti F. The top-down influence of ergogenic placebos on muscle work and fatigue. The European Journal of Neuroscience 2008 28(2):379-388.

Dourado E, Fernandes TCA, Meireles JSS, Monteiro IS. PAIN AND PLACEBO EFFECT. Revista de Cirurgia e Traumatologia Buco-Maxilo-Facial 2004 4(3):197-203.

MACHADO, Aryane Flauzino et al. Can Water Temperature and Immersion Time Influence the Effect of Cold Water Immersion on Muscle Soreness? A Systematic Review and Meta-Analysis. 
Sports Med, [s.I.], v. 46, n. 4, p.503-514, 18 nov. 2015. Springer Science + Business Media. http://dx.doi.org/10.1007/s40279-015-0431-7.

Broatch JR, Petersen A, Bishop DJ. Postexercise cold-water immersion benefits are not greater than the placebo effect. Med Sci Sports Exerc. 2014; 46(11):2139-47. https://doi.org/10.1249/MSS.0000000000000348.

World Health Organization. Obesity: Preventing and managing the global epidemic. Report of a World Health Organization Consultation. Geneva: World Health Organization. WHO Obesity Technical Report Series, n 284, p 256, 2000.

Sociedade Brasileira de Cardiologia / Sociedade Brasileira de Hipertensão / Sociedade Brasileira de Nefrologia. VI Diretrizes Brasileiras de Hipertensão. Arq Bras Cardiol. 2010; 95(1 supl.1):1-51

Baroni BM, Leal Junior EC, De Marchi T, Lopes AL, Salvador M, Vaz MA. Low level laser therapy before eccentric exercise reduces muscle damage markers in humans. Eur J Appl Physiol. 2010; 110(4):789-96. https://doi.org/10.1007/s00421-010-1562-z. 